'Universidad de Concepción. Departamento de Psiquiatría y Salud Mental. Facultad de Medicina, Universidad de Concepción. Concepción, Chile. ${ }^{2}$ Instituto de Ciencias Humanas Aplicadas (ICHA). Facultad de Psicología, Universidad San Sebastián. Santiago, Chile. apsicólogo.

${ }^{b}$ Doctorado en Salud Mental. CPhD.

Financiamiento: Esta investigación contó con financiamiento Proyecto interno Universidad San Sebastián No 505/2015. Proyecto CONICYT/ FONDECYT de iniciación No 11150852

Los autores declaran no tener conflictos de interés.

Recibido el 20 de marzo de 2019, aceptado el 30 de octubre de

Correspondencia a: Dr. Benjamín Vicente. Maipú № 2120, Concepción bvicent@udec.cl

\section{Recuperación de peso perdido en pacientes que han tenido cirugía bariátrica: Una mirada psicológica}

\author{
CARLA UGARTE ${ }^{1,2, a, b}$, ÁLVARO QUIÑONES ${ }^{2, a, c}$, BENJAMÍN VICENTE $^{1, \mathrm{c}}$
}

\section{Psychological factors influencing weight regain after bariatric surgery}

Background: Approximately 15 to $40 \%$ of bariatric patients regain a significant percentage of their weight lost after surgery. Among psychological variables, control and self-efficacy loci are associated with behaviors related to weight loss. Also, family support can be a risk or protection factor for the maintenance of weight loss. Aim: To evaluate the association between psychological variables with weight maintenance or regain after bariatric surgery. Material and Methods: We evaluated 97 patients at $4.1 \pm 3.4$ years after their bariatric surgery. They answered questionnaires about self-efficacy to lose weight, locus of weight control and family functioning style. Regain after surgery was also calculated, through self-report. Results: Seventeen percent of patients gained weight. Locus of control $(Z=-3.09, p<0.01)$, family identity $(Z=-3.71, p<0.01)$ and self-efficacy $(Z=-2.44, p=0.01)$, differentiated patients who maintained weight loss from those who re-gained at least $15 \%$ of their lost weight. An inverse and significant relationship was observed between the percentage of weight regain and locus of control $(r=-0.42, p<0.01)$, family identity $(r=-0.36, p<0.01)$ and self-efficacy $(r=-0.34, p<0.01)$. Conclusions: The psychological variables "locus of weight control" and "family identity" are inversely and moderately associated with weight regain in patients subjected to bariatric surgery.

(Rev Med Chile 2019; 147: 1390-1397)

Key words: Bariatric Surgery; Internal-External Control; Self Efficacy; Weight Loss.
L a cirugía bariátrica es actualmente el tratamiento más eficaz para tratar la obesidad, y indicada para personas con un índice cor igual o mayor a $40 \%$ o igual o mayor a $35 \%$ con comorbilidades ${ }^{1-3}$. Más específicamente, la cirugía permite una acelerada pérdida ponderal ${ }^{4}$. No obstante, un porcentaje no menor de pacientes recupera parte del exceso de peso perdido en el tiempo ${ }^{5-7}$.

La recuperación del peso postcirugía bariátrica ha sido definida como la recuperación de $15 \%$ o más de la pérdida del peso desde el nadir ${ }^{8-10}$ (menor peso registrado después de la cirugía).
La recuperación del peso es un fenómeno multifactorial y complejo ${ }^{11}$. Aproximadamente, entre 15 y $40 \%$ de los pacientes sometidos a cirugía bariátrica recuperan un porcentaje significativo del peso perdido en el mediano o largo plazo. La recuperación del peso incluso se ha informado en el corto plazo, próxima al momento del alta ${ }^{12}$.

Para evaluar el porcentaje de recuperación del peso se utilizó el porcentaje de peso total perdido $(\% \mathrm{PTP})^{8,13}$. Este indicador permite comparar series de pacientes sin el sesgo del IMC inicial.

En el proceso de recuperación del peso en pacientes sometidos a cirugía bariátrica influyen 
variables de diversa naturaleza, entre las que destacan variables psicológicas ${ }^{14,15}$ (autoeficacia, perspectiva temporal, apoyo familiar, entre otras) y conductuales ${ }^{16}$ (estilo de vida, alimentación y ejercicio).

En el estudio de los predictores de la recuperación de peso $^{17,18}$, desde hace algunos años se han investigado variables psicológicas de tipo cognitivo-actitudinal como la autoeficacia, el locus de control y el funcionamiento familiar, específicamente, su relación con la adherencia a cambios en el estilo de vida para mantener la pérdida de peso ${ }^{19-21}$.

La variable psicológica "autoeficacia" fue desarrollada por la teoría del aprendizaje social de Albert Bandura (1977) y se define como los juicios que cada persona hace acerca de sus capacidades para lograr un resultado deseado ${ }^{22,23}$. Por su parte, la autoeficacia percibida para el control del peso se refiere a las creencias acerca de las capacidades personales para organizar y ejecutar los cursos de acción necesarios para controlar el peso corporal ${ }^{24}$. Diversos estudios ${ }^{25-27}$ han examinado su capacidad para predecir el éxito, tanto para la pérdida de peso como para su mantenimiento. Otros estudios se han enfocado en la capacidad para predecir la intención de realizar conductas en torno a una alimentación saludable y la realización de ejercicio físico $^{26}$. La autoeficacia, también, se ha asociado positivamente con la pérdida del peso al inicio de los tratamientos ${ }^{28}$. Así, se sostiene que el éxito en tratamientos para la pérdida del peso debiese mejorar la autoeficacia en la alimentación y regulación del peso y que esta mejora en la percepción de autoeficacia puede predecir el mantenimiento exitoso del peso perdido a largo plazo ${ }^{29}$.

Batsis et al. ${ }^{29}$ examinaron la relación entre la pérdida de peso y la autoeficacia en pacientes con cirugía bariátrica y encontraron que la pérdida de peso producto de la cirugía estuvo asociada a mayores puntuaciones en la autoeficacia alimentaria. Además, encontraron que la asociación de esta relación suele disminuir con el tiempo y que ello podría ayudar a explicar, en parte, la recuperación de peso que a largo plazo presentan algunos pacientes sometidos a cirugía bariátrica.

La variable psicológica "locus de control" 30 (LC), introducida por la teoría del aprendizaje social de Rotter, se refiere a las creencias personales acerca del grado de control que se tiene sobre los acontecimientos vitales. Rotter ${ }^{30}$ diferencia dos tipos de locus de control: interno (LCI) y externo (LCE). El primero hace referencia a la creencia de que los acontecimientos son contingentes a la conducta de la persona, y el segundo se refiere a la creencia que estos acontecimientos no son contingentes a las propias acciones, sino que dependen de la suerte, la casualidad, el destino o el poder de los demás.

Particularmente, la investigación sobre locus de control y obesidad indica una relación positiva entre el LCI y completar con éxito un programa de pérdida de peso $^{31}$. El LCI también se ha asociado a la mantención de la pérdida de $10 \%$ del peso corporal en personas con sobrepeso ${ }^{21}$. Asimismo, en pacientes con sobrepeso u obesidad que presentan un LCI, se observa que la motivación para la pérdida de peso aumenta luego de leer material de educación para la salud. En cambio, esta relación no se ha observado en personas con $\mathrm{LCE}^{32}$. De igual modo existe relación entre LCI, alta autoestima y una mayor adherencia a la dieta y el ejercicio ${ }^{33}$.

Por otra parte, la interrupción del tratamiento o del seguimiento después de lograr una pérdida de peso en personas que presentan un LCE, conduce progresivamente a la recuperación del mismo ${ }^{22}$.

El apoyo familiar es una variable psicológica que se ha asociado a la mantención y recuperación del peso postcirugía bariátrica ${ }^{14}$. Es conocido que la cirugía bariátrica por sí misma tiene buenos resultados en términos de pérdida de peso, pero para mantener estos resultados en el tiempo se requieren cambios en el estilo de vida. En este contexto, hay estudios ${ }^{34,35}$ que han informado que la familia puede ser un factor protector y facilitador para el éxito del tratamiento quirúrgico o un factor de riesgo cuando se ingiere alimentos calóricos en la dieta cotidiana.

En el presente estudio se plantearon las siguientes dos hipótesis:

1. Los pacientes que mantengan la pérdida de peso se diferenciarán significativamente de los que recuperen $15 \%$ o más del peso total perdido en autoeficacia, locus de control y compromiso familiar, incluso al controlar por tiempo postoperatorio.

2. A mayor recuperación de peso se observarán menores niveles de autoeficacia, un locus de control más externo y menores niveles de compromiso familiar.

\section{Objetivo}


Evaluar la asociación entre las variables psicológicas "autoeficacia para bajar de peso", "locus de control de comer y engordar" e "identidad familiar” con la mantención y la recuperación de peso postcirugía bariátrica.

\section{Material y Método}

Se realizó un estudio de corte transversal y alcance correlacional con 97 pacientes que habían sido sometidos a cirugía bariátrica a través de un muestro no probabilístico por conveniencia. Los criterios de exclusión fueron: pacientes menores de 18 años; técnica de intervención distinta a bypass gástrico o gastrectomía vertical; pacientes con cirugía revisional o de conversión; mujeres que se encontrasen en gestación o período de lactancia.

Los pacientes respondieron en forma autoadministrada un conjunto de cuestionarios que incluía datos sociodemográficos, las escalas "autoeficacia para bajar de peso"36, "locus de control frente a la conducta de comer y engordar" 37 , y la dimensión "identidad familiar" de la escala "estilo de funcionamiento familiar"38 (Véase Cuadro 1). También autorreportaron su estatura, su peso prequirúrgico, peso nadir y peso al momento del estudio. Es de destacar que los datos antropométricos autorreportados (peso y talla) han sido considerados como variables de resultado fiable en pacientes sometidos a cirugía bariátrica ${ }^{39}$.

\section{Variables de resultado}

Recuperación del \%PTP: Corresponde al porcentaje de recuperación de peso entre el nadir y el momento del estudio. Esta variable se analizó como variable numérica y como variable categórica a través de ecuaciones que figuran en el Cuadro 2. El cálculo del \%PTP se obtuvo utilizando los datos del autorreporte de los pesos de los pacientes.

Ética: La investigación fue revisada y aprobada por el Comité de Ética de la Universidad de Concepción, y el Comité de Ética Científica del Servicio de Salud de Concepción.

\section{Análisis estadístico}

Se utilizó la prueba $U$ de Mann Whitney para estudiar la diferencia entre los pacientes que recuperan versus los que mantiene el \%PTP_nadir y las variables psicológicas. Para calcular el tamaño del efecto de las diferencias se utilizó $r$ de Rosenthal; y el coeficiente de correlación de Pearson para determinar la relación entre las variables. Para controlar el efecto del tiempo postoperatorio se utilizó ANCOVA y correlaciones parciales.

\section{Cuadro 1. Características de los instrumentos psicométricos utilizados}

\begin{tabular}{|c|c|c|c|c|c|}
\hline Instrumento & Tipo de escala & $\begin{array}{l}\text { Dimensiones evaluadas } \\
\text { en la investigación }\end{array}$ & $\begin{array}{c}\alpha \text { de } \\
\text { Cronbach }\end{array}$ & $\begin{array}{l}\text { Tipo de } \\
\text { validez }\end{array}$ & $\begin{array}{l}\text { Interpretación } \\
\text { de puntajes }\end{array}$ \\
\hline \multirow{3}{*}{$\begin{array}{l}\text { Autoeficacia } \\
\text { para bajar de } \\
\text { peso }\end{array}$} & \multirow{3}{*}{$\begin{array}{l}\text { Escala Likert de } 7 \\
\text { puntos que va desde } \\
0 \text { (no puedo hacerlo) } \\
\text { a } 7 \text { (seguro/a de } \\
\text { poder hacerlo) }\end{array}$} & $\begin{array}{l}\text { Autoeficacia para hacer } \\
\text { dieta }\end{array}$ & 0,93 & \multirow[t]{3}{*}{ Constructo } & \multirow{3}{*}{$\begin{array}{l}\text { Mayores puntajes } \\
\text { implican mayor } \\
\text { percepción de } \\
\text { autoeficacia }\end{array}$} \\
\hline & & $\begin{array}{l}\text { Autoeficacia para hacer } \\
\text { ejercicio }\end{array}$ & 0,94 & & \\
\hline & & $\begin{array}{l}\text { Autoeficacia para comprar } \\
\text { alimentos }\end{array}$ & 0,93 & & \\
\hline \multirow{2}{*}{$\begin{array}{l}\text { Locus de } \\
\text { control frente } \\
\text { a la conducta } \\
\text { de comer y } \\
\text { engordar }\end{array}$} & \multirow{2}{*}{$\begin{array}{l}\text { Escala Likert de } \\
4 \text { puntos que va } \\
\text { desde } 1 \text { (totalmente } \\
\text { en desacuerdo) a } \\
4 \text { (totalmente de } \\
\text { acuerdo) }\end{array}$} & $\begin{array}{l}\text { Percepción de control } \\
\text { respecto de comer }\end{array}$ & 0,83 & \multirow[t]{2}{*}{$\begin{array}{l}\text { Contenido } \\
\text { Constructo }\end{array}$} & \multirow{2}{*}{$\begin{array}{l}\text { Mayores puntajes } \\
\text { implican } \\
\text { una mayor } \\
\text { percepción de } \\
\text { control o un } \\
\text { locus de control } \\
\text { más interno }\end{array}$} \\
\hline & & $\begin{array}{l}\text { Percepción de control } \\
\text { respecto de engordar y } \\
\text { adelgazar }\end{array}$ & & & \\
\hline $\begin{array}{l}\text { Estilo de } \\
\text { funcionamiento } \\
\text { familiar }\end{array}$ & $\begin{array}{l}\text { Escala Likert de } 5 \\
\text { puntos que va desde } \\
1 \text { (casi nunca) a } 5 \\
\text { (casi siempre) }\end{array}$ & Identidad familiar & 0,79 & $\begin{array}{l}\text { Contenido } \\
\text { Constructo }\end{array}$ & $\begin{array}{l}\text { Mayores puntajes } \\
\text { implican mayor } \\
\text { compromiso y } \\
\text { cohesión familiar }\end{array}$ \\
\hline
\end{tabular}




\section{Cuadro 2. Ecuaciones del \%PTP y operacionalización de las variables de resultado}

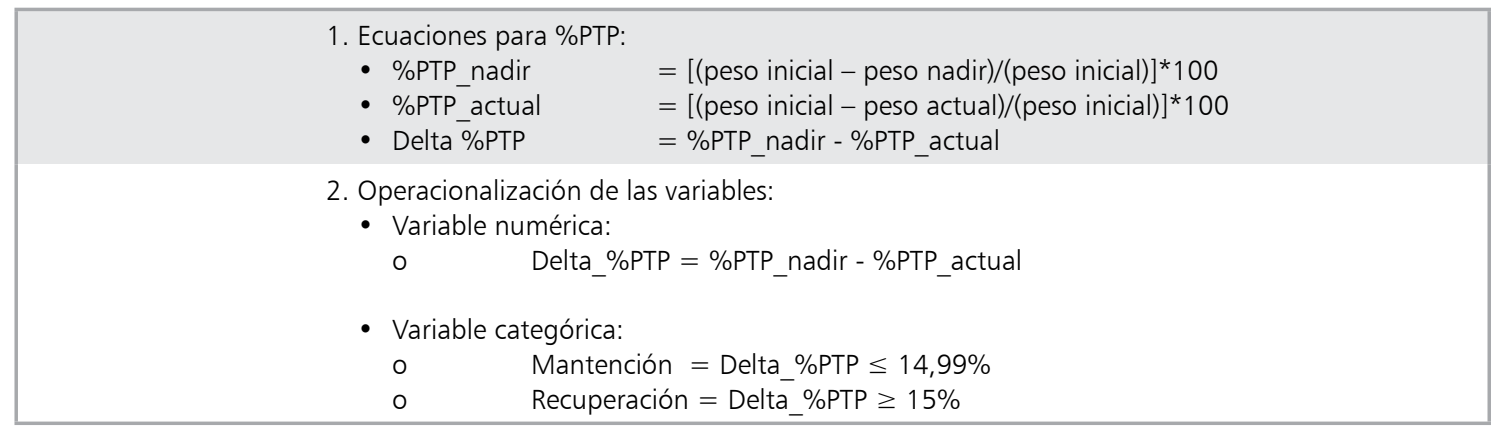

\%PTP: Porcentaje de peso total perdido. \%PTP_nadir: Porcentaje de peso total perdido en el nadir. \%PTP_actual: Porcentaje de peso total perdido al momento del estudio. Delta_\%PTP: Diferencia simple entre el porcentaje de peso total perdido en el nadir y en el momento del estudio.

Tabla 1. Evolución del estado nutricional de la muestra

\begin{tabular}{|lcccccc|}
\hline Momento & Normopeso & Sobrepeso & $\begin{array}{c}\text { Estado nutricional } \\
\text { Obesidad I }\end{array}$ & $\begin{array}{c}\text { Obesidad II } \\
\text { Obesidad III }\end{array}$ & Obesidad IV \\
\hline Previo & - & $2,1 \%$ & $16,5 \%$ & $27,8 \%$ & $43,3 \%$ & $10,3 \%$ \\
Nadir & $49,5 \%$ & $36,1 \%$ & $9,3 \%$ & $3,1 \%$ & $2,1 \%$ & - \\
Actual & $36,1 \%$ & $37,1 \%$ & $18,6 \%$ & $3,1 \%$ & $5,2 \%$ & - \\
\hline
\end{tabular}

\section{Resultados}

De los 97 pacientes que participaron en este estudio, solo 10,3\% $(\mathrm{n}=10)$ eran hombres. La edad de la muestra estuvo comprendida entre los 20 y 66 años, con un promedio de 39,78 años (DE 10,25). Al momento del estudio, el promedio de años postoperatorios fue de $4,1(\mathrm{DE}=3,4)$.

En relación a la evolución del estado nutricional (Tabla 1) previo a la cirugía, $97,9 \%$ de la muestra presentó algún grado de obesidad. En el nadir, $14,5 \%$ de los pacientes mantuvo un estado nutricional de obesidad, porcentaje que subió a $26,9 \%$ al momento del estudio.

La diferencia entre los promedios de los \%PTP en el nadir y en el momento actual fue de 7,98 puntos (Tabla 2).

Desde el nadir al momento del estudio $17 \%$ de los pacientes presentó recuperación del \%PTP, definida como la recuperación de $15 \%$ o más del peso perdido.

En relación a las variables psicológicas (Tabla 3), se observó una diferencia significativa entre los pacientes que mantenían o recuperaban peso y el
Tabla 2. Descriptivos del porcentaje de peso total perdido en el nadir y momento de evaluación

\begin{tabular}{|lccccc|}
\hline Momento & n & M & $\begin{array}{c}\text { \%PTP } \\
\text { DE }\end{array}$ & Mín & Máx \\
Nadir & 97 & 39,59 & 8,96 & 16 & 59 \\
Actual & 97 & 31,61 & 10,43 & 2 & 55 \\
\hline
\end{tabular}

\%PTP: Porcentaje de peso total perdido.

locus de control frente a la conducta de comer y engordar $(Z=-3,09 ; p<0,002 ; r=-0,31)$. Más específicamente, se encontró diferencias entre los pacientes que mantuvieron el \%PTP y los que lo recuperaron en "percepción de control respecto a comer" $(Z=-3,32 ; p<0,001)$ y "percepción de control respecto engordar y adelgazar" $(Z=-2,96$; $p=0,003)$. El tamaño de efecto fue de $r=-0,34 y$ de $r=-0,30$ (efecto pequeño), respectivamente.

En suma, los pacientes que mantuvieron la pérdida de peso presentaron mayores puntajes en percepción de control "respecto a comer", "a engordar y adelgazar" y "locus de control frente a la conducta de comer y engordar". 
Tabla 3. Relación entre la mantención y recuperación del porcentaje de peso total perdido y variables psicológicas

\begin{tabular}{|c|c|c|c|c|}
\hline Variable psicológica & $\begin{array}{l}\text { Mantención } \\
\text { Med (DE) }\end{array}$ & $\begin{array}{l}\text { Recuperación } \\
\text { Med (DE) }\end{array}$ & $Z(p)$ & r de Rosenthal \\
\hline Autoeficacia para bajar de peso & $168 \quad(34,7)$ & $(37,2)$ & $-2,44(0,015)$ & $-0,25$ \\
\hline Para hacer dieta & $69 \quad(15,2)$ & $(17,0)$ & $-2,41(0,016)$ & $-0,24$ \\
\hline Para hacer ejercicio & $57 \quad(19,8)$ & $41 \quad(17,5)$ & $-2,36(0,018)$ & $-0,23$ \\
\hline Para comprar alimentos & $(8,7)$ & $(9,3)$ & $-1,43(0,15)$ & \\
\hline Locus de control general del peso & $132,5(16,2)$ & $113,4(22,0)$ & $-3,09(0,002)$ & $-0,31$ \\
\hline Para comer & $48,2(6,5)$ & $40,4(8,5)$ & $-3,32(0,001)$ & $-0,34$ \\
\hline Para adelgazar y engordar & $84,2(10,8)$ & $72,9(16,2)$ & $-2,96(0,003)$ & $-0,30$ \\
\hline $\begin{array}{l}\text { Funcionamiento familiar } \\
\text { Identidad familiar }\end{array}$ & $(8,2)$ & $(10,3)$ & $-3,71(0,000)$ & $-0,38$ \\
\hline
\end{tabular}

Por otra parte, en relación a la "autoeficacia para bajar de peso" y sus tres subhabilidades, se observó una diferencia significativa entre los pacientes que mantuvieron el \%PTP o lo recuperaron en dos subhabilidades "para hacer dieta" $(Z=-2,41 ; p=0,016 ; r=-0,24)$ y "para hacer ejercicio" $(Z=-2,36 ; p=0,018 ; r=-0,23)$. También, la "autoeficacia general para bajar de peso" permitió diferenciar significativamente a los mantenedores de los recuperadores del peso $(Z=-3,10 ; p<0,01 ; r=-0,25)$. En definitiva, los pacientes que presentaron recuperación del \%PTP presentaron menores niveles de "autoeficacia para bajar de peso", "para hacer dieta” y "para hacer ejercicio".

Por otro lado, la variable identidad familiar también diferenció significativamente a los pacientes que mantuvieron el \%PTP de los que lo recuperaron $(Z=-3,71 ; p<0,001 ; r=-0,38)$. Específicamente, tuvieron menores puntuaciones en identidad familiar los pacientes que presentaron recuperación del \%PTP.

Ahora bien, al controlar por tiempos postoperatorios se encontró que "identidad familiar" $(\mathrm{F}=8,71 ; \mathrm{p}=0,004 ; \eta 2=0,085)$, "percepción de control respecto a comer" ( $\mathrm{F}=11,47 ; \mathrm{p}=0,001$; $\eta 2=0,10)$, "respecto a engordar y adelgazar" $(F=8,97 ; p=0,003 ; \eta 2=0,087)$ y "locus de control frente a la conducta de comer y engordar" $(\mathrm{F}=11,10 ; \mathrm{p}=0,001 ; \eta 2=0,10)$ permitieron diferenciar significativamente a los pacientes que recuperan \%PTP de los que mantienen la pérdida del peso. Ninguna de las dimensiones de la autoeficacia resultaron significativas para diferenciar a los grupos cuando se controló por tiempo postoperatorio.

En otro orden de cosas, se evaluó el grado y tipo de relación entre la variable de resultado numérica y las variables psicológicas. Se utilizó el coeficiente de correlación producto momento de Pearson. En último lugar, al controlar el efecto del tiempo postoperatorio solo las relaciones entre la recuperación del peso y las subhabilidades "autoeficacia para hacer dieta" y "autoeficacia para comprar alimentos saludables" dejaron de ser significativas. Los resultados se presentan en la Tabla 4.

\section{Discusión}

En primer lugar, $17 \%$ de los pacientes presentaron recuperación de peso de $15 \%$ o más del \%PTP desde el nadir. Estos resultados son coherentes con los porcentajes de recuperación informados por los estudios en pacientes sometidos a cirugía bariátrica ${ }^{5,7}$.

En segundo lugar, los resultados confirman parcialmente las dos hipótesis planteadas:

La primera hipótesis se cumple parcialmente, es decir, las variables psicológicas evaluadas permiten diferenciar a los pacientes que mantienen 
Tabla 4. Correlaciones entre las variables psicológicas y el porcentaje de recuperación

\begin{tabular}{|lcc|}
\hline & Correlación entre variables & $\begin{array}{c}\text { Correlación controlando por } \\
\text { tiempo postoperatorio }\end{array}$ \\
\hline 1. Recuperación del \%PTP & 1 & 1 \\
\hline 2. Autoeficacia para hacer dieta & $-0,289^{* *}$ & $-0,183$ \\
\hline 3. Autoeficacia para hacer ejercicio & $-0,327^{* *}$ & $-0,221^{*}$ \\
\hline 4. Autoeficacia para comprar alimentos & $-0,208^{*}$ & $-0,018$ \\
\hline 5. Autoeficacia escala total & $-0,316^{* *}$ & $-0,198$ \\
\hline 6. Identidad familiar & $-0,361^{* *}$ & $-0,260^{*}$ \\
\hline 7. Locus de control comer & $-0,416^{* *}$ & $-0,333^{* *}$ \\
\hline 8. Locus de control adelgazar engordar & $-0,397^{* *}$ & $-0,324^{* *}$ \\
\hline 9. Locus de control escala total & $-0,424^{* *}$ & $-0,345^{* *}$ \\
\hline
\end{tabular}

\%PTP: Porcentaje de peso total perdido. ${ }^{*} p<0,05 ;{ }^{*} p<0,01$.

el \%PTP de los que lo recuperan. Sin embargo, cuando se controla por tiempo postoperatorio la variable autoeficacia y sus tres subhabilidades dejan de ser significativas, por lo que esta hipótesis se confirma para las variables "locus de control" e "identidad familiar", pero no para la variable "autoeficacia".

Es de destacar que se esperaba que la variable psicológica "autoeficacia para bajar de peso" diferenciase significativamente a los pacientes que mantienen la pérdida de peso versus los que lo recuperan, independiente del tiempo postoperatorio presentado. En definitiva, estos resultados indican que es más relevante para la mantención de la pérdida del peso que el paciente considere que esta mantención depende principalmente de su comportamiento, a la valoración que pueda tener de estar realizando bien o mal una serie de conductas para mantener la pérdida del peso (autoeficacia).

La segunda hipótesis se cumple para todas las variables psicológicas, las que se asocian de forma inversa y significativa a la recuperación del peso desde el nadir. Es decir, a mayor recuperación menores niveles de locus de control, identidad familiar y autoeficacia. No obstante, al controlar por tiempo postoperatorio las relaciones se mantienen significativas para las variables "locus de control" e "identidad familiar". En relación a la variable autoeficacia, se mantiene una asociación significativa solo con la dimensión "para hacer ejercicio". Esto último es coherente con los estudios que indican que el ejercicio físico en más relevante en la mantención de la pérdida de peso ${ }^{40}$ que en la pérdida misma.

En suma, un locus de control interno y un fuerte compromiso familiar son factores protectores para la mantención del peso perdido postcirugía.

Las limitaciones de la presente investigación aluden a la variabilidad de los tiempos postoperatorios y que la muestra estuvo conformada principalmente por mujeres $(89,7 \%)$. Una reflexión que se desea explicitar, es que la presente investigación, con las limitaciones destacadas, entrega información que aporta para conocer y comprender la influencia de las variables psicológicas evaluadas en la evolución del peso en pacientes bariátricos.

\section{Investigaciones futuras}

Para avanzar en la comprensión de las variables que se encuentran implicadas en la recuperación del peso, podría aportar la inclusión de variables relacionadas con el estilo de vida, a través de estudios de correlación, regresión y mediación. También, la inclusión de otros constructos psicológicos relacionados, por ejemplo regulación emocional, abuso de alcohol o sustancias, comer emocional y trastorno por atracones.

Otra área interesante a explorar, y que puede ser complementaria a la investigación presente, es la prevención de la recuperación del peso. Para ello es posible explorar la influencia de las variables contextuales, como la adherencia al seguimiento por parte del equipo de intervención y la participación en grupos de pacientes sometidos a cirugía bariátrica. 


\section{Conclusión}

Estos resultados confirman que dos de las variables psicológicas evaluadas se asocian a la mantención y recuperación de peso en pacientes sometidos a cirugía bariátrica. Particularmente, el locus de control y las atribuciones internas tienen relevancia asociativa en la mantención de la pérdida del peso. En este contexto, la asociación encontrada entre la mantención del peso y las variables psicológicas analizadas da información acerca de la importancia de implicar activamente a los pacientes en su propio proceso y también a sus familias. Profundizar en el estudio de estas y otras variables puede entregarnos información relevante para el desarrollo de programas de prevención de la recuperación de peso en pacientes sometidos a cirugía bariátrica. En coherencia con lo anterior, es de la mayor relevancia para obtener buenos resultados y mantenerlos ayudar a los pacientes a entender mejor que son "protagonistas y espectadores de su propia obra" en la reducción del exceso de peso y su mantención.

Agradecimientos: A todos los pacientes que participaron voluntariamente del presente estudio. Especialmente le agradezco al Dr. Gerardo Astete (Q.E.P.D) por su ayuda para la realización de la presente investigación y a los psicólogos Fernanda Mena y Jorge Figueroa del Equipo de Tratamiento Quirúrgico para la Obesidad de Concepción (ETO-Conce).

\section{Referencias}

1. Courcoulas A, King W, Belle S, Berk P, Flum D, Garcia L, et al. Seven-Year Weight Trajectories and Health Outcomes in the Longitudinal Assessment of Bariatric Surgery (LABS) Study. JAMA Surg 2018; 153 (5): 427-34.

2. Sabench F, Domíngez-Adame E, Ibarzabal A, Socas M, Valentí V, García A, et al. Criterios de calidad en cirugía bariátrica: revisión de conjunto y recomendaciones de la Asociación Española de Cirujanos y de la Sociedad Española de Cirugía de la Obesidad. Cir Esp 2017; 95 : 4-16.

3. Wolfe B, Kvach E, Eckel R. Treatment of Obesity: Weight Loss and Bariatric Surgery. Circ Res 2016; 27; 118(11): 1844-55.

4. Adams T, Davidson L, Litwin S, Kim J, Kolotkin R, Nan- jee $\mathrm{N}$, et al. Weight and metabolic outcomes 12 years after Gastric Bypass. N Engl J Med 2017; 377: 1143-55.

5. Jiménez L, Mendoca F, Mendoza F, Pimentel M, Gestic $\mathrm{M}$, Chaim E, et al. Impact of Weight Regain on the Evolution of Non-alcoholic Fatty Liver Disease After Roux-en-Y Gastric Bypass: a 3-Year Follow-up. Obesity Surgery 2018; 28 (10): 3131-5.

6. Clapp B, Wynn M, Martyn C, Foster C, O’Dell M, Tyroch A. Long term (7 or more years) outcomes of the sleeve gastrectomy: a meta- analysis. Surg Obes Relat Dis 2018; 14 (6): 741-7.

7. Sheppard C. Lester E. Chuck A. Birch D. Karmali S. Gara C. The Economic Impact of Weight Regain. Gastroenterol Res Pract 2013. http://dx.doi. org/10.1155/2013/379564.

8. Shukla A, He D, Saunders K, Andrew C, Aronne L. Current concepts in management of weight regain following bariatric surgery. Expert Rev Endocrinol Metab 2018; 13 (2): 67-76.

9. Cooper T, Simmons E, Webb K, Burns J, Kushner R. Trends in weight regain following Roux-en-Y Gastic Bypass (RYGB) Bariatric Surgery. Obes Surg 2015; 25 : 1474-81.

10. Odom J, Zalesin KC, Washington TL, Miller WW, Hakmeh B, Zaremba DL, et al. Behavioral predictors of weight regain after bariatric surgery. Obes Surg 2010; 20 (3): 349-56.

11. Maleckas A, Gudaityté R, Petereit R, Venclauskas L, Velickiené $\mathrm{D}$. Weight regain after gastric bypass: etiology and treatment options. Glan Surg 2016; 5 (6): 617-24.

12. Lemanu DP, Singh PP, Rahman H, Hill AG, Babor R, MacCormick AD. Five-year results after laparoscopic sleeve gastrectomy: a prospective study. Surg Obes Relat Dis 2015; 11: 518-24.

13. Van de Laar A, van Rijswijk A, Kakas H, Bruin S. Sensitivity and Specificity of 50\% Excess Weight Loss (50\%EWL) and Twelve Other Bariatric Criteria for Weight Loss Success. Obesity Surgery 2018; 28 (8): 2297-304.

14. Schiavo L, Scalera G, Pilone V, De Sena G, Ciorra FR, Barbarisi A. Patient adherence in following a prescribed diet and micronutrient supplements after laparoscopic sleeve gastrectomy: our experience during 1 year of follow-up. J Hum Nutr Diet 2017; doi: 10.1111/jhn.12427.

15. Canetti L, Berry E, Elizur Y. Psychosocial predictors of weight loss and psychological adjustment following bariatric surgery and a weight-loss program: The mediating role of emotional eating. Int J Eat Disord 2009; 42 (2): 109-17.

16. Wakayama L, Nameth K, Adler S, Safer D. Replication and extension of dietary adherence as a predictor of su- 
boptimal weight-loss outcomes in postbariatric patients. Surg Obes Relat Dis 2019; 15 (1): 91-6.

17. Papapietro K. [Weight gain after bariatric surgery]. Rev Chil Cir 2012; 64 (1): 83-7.

18. Wedin S, Madan A, Correll J, Crowley N, Malcolm R, Byrned $\mathrm{K}$, et al. Emotional eating, marital status and history of physical abuse predict 2-year weight loss in weight loss surgery patients. Eat Behav 2014; 15 (4): 619-24.

19. Peterhänsel C, Linde K, Wagner B, Dietrich A, Kersting A. Subtypes of Personality and 'Locus of Control' in Bariatric Patients and their Effect on Weight Loss, Eating Disorder and Depressive Symptoms, and Quality of Life. Eur Eat Disord Rev 2017; 25 (5): 397-405.

20. Lent M, Bailey-Davis L, Irving B, Wood G, Cook A, Hirrsch A. Franceschelli-Hosterman J. Bariatric surgery patients and their families: Health, Psysical activity, and social support. Obes Surg 2016; 26: 2981-8.

21. Nickel F, Schmidt L, Bruckner T, Büchler MW, Müller-Stich B, Fischer L. Influence of bariatric surgery on quality of life, body image, and general self-efficacy within 6 and 24 months- a prospective cohort study. Surg Obes Relat Dis 2017; 13 (2): 313-9.

22. Anastasiou C, Fappa E, Karfopoulou E, Gkza A, Yannakoulia $\mathrm{M}$. Weight loss maintenance in relation to locus of control: The MedWeight study. Behav Res Ther 2015; 71: 40-4.

23. Bandura A. Human agency in social cognitive theory. Am Psychol 1989; 44: 1175-84.

24. González M, Lugli Z. Control personal de la conducta y adhesión terapéutica en balón intragástrico Bioenterics. Gen 2012; 66 (4).

25. Menéndez-González L, Orts-Cortés M. Psychosocial and behavioural factors in the regulation of weight: Self-regulation, self-efficacy and locus control. Enferm Clin 2018; 28 (3): 154-61.

26. Campos Y, Argüelles V, Vázquez F, Ortiz M. Autorregulación, autoeficacia y orientación nutricional para la disminución del exceso de peso. Revista Argentina de Clínica Psicológica 2014; XXIII (2): 163-70.

27. Latner J, McLeod G, O'Brien K, Johnston L. The role of self-efficacy, coping, and lapses in weight maintenance. Eat Weight Disord 2013; 18 (4): 359-66.

28. Choo J, Kang H. Predictors of initial weight loss among women with abdominal obesity: a path model using self-efficacy and health-promoting behavior. J Advan Nurs 2015; 71 (5): 1087-97.

29. Batsis J, Clark M, Grothe K, Lopez-Jimenez F, Collazo-Clavell M, Somers V, et al. Self-efficacy after bariatric surgery for obesity. A population-based cohort study. Appetite 2009; 52 (3): 637-45.

30. Rotter J. Generalized expectancies for internal versus external control of reinforcement. Psychol Monogr 1966; 80 (1): 1-28.

31. Balch P, Ross AW. Predicting sucess in weight reduction as a function of locus of control: a unidimensional and multidimensional approach. J Consult Clin Psych 1975; 43: 119.

32. Holt CL, Clark EM, Kreuter MW. Weight locus of control and weight-related attitudes and behaviors in an overweight population. Addict Behav 2001; 26: 329e340.

33. Donovan C, Chew D, Penny R. Perfecting Weight Restriction: The Moderating Influence of Body Dissatisfaction on the Relationship Between Perfectionism and Weight Control Practices. Behav Change 2014; 31 (03): 189-204.

34. Cruzat-Mandich C, Díaz-Castrillón F, García A, Ulloa V. Cirugía bariátrica en pacientes jóvenes: ¿Cómo evalúan el proceso? Rev Chil Nutr 2018; 45 (1): 17-27.

35. Moore DD, Cooper CE. Life after bariatric surgery: Perceptions of male patients and their intimate relationships. J Marit Fam Ther 2016; 42: 495-508.

36. Campos S, Pérez J. Autoeficacia y conflicto decisional frente a la disminución del peso corporal en mujeres. Rev Chil Nutr 2007; 34 (3): 213-8.

37. Marín L. Construcción y validación de un instrumento de evaluación de locus de control aplicado a pacientes obesos que buscan tratamiento médico convencional y tratamiento quirúrgico para bajar de peso [Internet]. Santiago, Chile: Universidad de Chile - Facultad de Ciencias Sociales; 2007 [citado: 2019, julio]. Disponible en: http://repositorio.uchile. $\mathrm{cl} /$ handle/2250/106596.

38. Larraín M, Zegers B, Díez I, Trapp A, Polaino-Lorente A. Validez y Confiabilidad de la Versión Española de la Escala del Estilo de Funcionamiento Familiar (EFF) de Dunst, Trivette \& Deal para el Diagnóstico del Funcionamiento Familiar en la Población Chilena. Psykhe 2011; 12 (1).

39. Gokler M. Bugrul N. Sari A. Metintas S. The validity of self-reported vs. measured body weight and height and the effect of self-perception. Arch Med Sci 2018; 14, 1: 174-81.

40. Rothwell L, Kow L, Toouli J. Effect of a Post-operative Structured Exercise Programme on Short-Term Weight Loss After Obesity Surgery Using Adjustable Gastric Bands. Obes Surg 2015; 25 (1): 126-28. 